\title{
The Witten-Sakai-Sugimoto model: A brief review and some recent results
}

\author{
Anton Rebhan ${ }^{1, a}$ \\ ${ }^{1}$ Institute for Theoretical Physics, TU Wien \\ Wiedner Hauptstr. 8-10, A-1040 Vienna, Austria
}

\begin{abstract}
A brief review of the Witten-Sakai-Sugimoto model is given, which is a topdown holographic model of low-energy QCD with chiral quarks derived from type-IIA superstring theory. The main predictions of the model, in particular concerning meson spectra, the gluon condensate, the QCD string tension, the mass of the $\eta^{\prime}$ and of baryons are discussed and compared quantitatively with available experimental and/or lattice results. Then some recent results of potential interest to the physics program at the future FAIR facility are presented: The spectrum of glueballs and their decay rates into pions, and the phase diagram of QCD at finite temperature, density, and magnetic field strength.
\end{abstract}

\section{Introduction}

Quantum chromodynamics (QCD) is the well-established theory of the strong nuclear forces. Thanks to asymptotic freedom, it is amenable to perturbative methods at high energy. While the coupling never becomes very small at any energy scale of interest, perturbative QCD is a most successful framework for collider physics, and its predictions beyond the simple parton model have been tested extensively. Nonperturbative aspects of QCD, such as the spectrum of hadrons and the transition to a quark-gluon plasma phase, can be studied from first principles through lattice QCD. Indeed, tremendous progress has been made thanks to the availability of powerful computers and also thanks to methodological progress. However, two areas of strong-coupling physics, where lattice QCD is still far from giving reliable answers are finite baryon density and real-time phenomena such as decay rates (although progress is being made on both frontiers). These insufficiently charted areas will be at the focus of a new generation of experiments at the FAIR facility, where the PANDA experiment at FAIR will study hadron reactions $[1,2]$, and the CBM experiment will aim at exploring baryonic matter at high density [3].

A novel approach to strongly coupled gauge theories has been developed over the last one and a half decades in the form of gauge/gravity duality [4], also known as "holography". While firstprinciples approaches with a well-defined string-theoretic foundation allow one to study only gauge theories which are in important ways different from real QCD, this approach offers fresh insights in the richness of phenomena of gauge theories at strong coupling. Based on this progress, so-called bottomup models have been developed which do not care so much about a string-theoretic justification, but

\footnotetext{
ae-mail: rebhana@tph.tuwien.ac.at
} 
provide phenomenological models that incorporate the key ingredients of holographic gauge theories, and many interesting studies have been carried out using these.

There is however one top-down holographic model, which goes a long way towards QCD starting from a fundamental string-theoretic basis: Witten's model of low-energy QCD through type-IIA supergravity, proposed already in 1998 [5], which has almost no free parameters. Here one sets up a holographic dual to a supersymmetric gauge theory in 4+1 dimensions and performs the analogue of high-temperature dimensional reduction, but with respect to the superfluous spatial dimensions, such that at low energy one ends up with nonsupersymmetric pure-glue QCD at large number of colors and large 't Hooft coupling in 3+1 dimensions.

In 2004, Sakai and Sugimoto [6, 7] have extended Witten's model by flavor D8-branes in a setup that provides chiral quarks and anti-quarks. In the model of Sakai and Sugimoto chiral symmetry breaking as well as its restoration at high temperatures has a beautiful geometric realization. Its low-energy effective action contains a chiral Lagrangian for massless Nambu-Goldstone bosons and massive vector and axial vector mesons, complete with the correct Wess-Zumino-Witten term and also a Skyrme term. The only free parameters in the model are a (Kaluza-Klein) mass parameter $M_{\mathrm{KK}}$ and the 't Hooft coupling $\lambda$ at this scale, making it very predictive. Unfortunately, hardly anything is known about the corrections that are needed to approach real QCD at finite $N_{c}$ and $\lambda$ and that are in principle determined by type-IIA string theory. In the case of the original AdS/CFT correspondence for maximally supersymmetric Yang-Mills theory based on type-IIB string theory, higher-derivative string-theoretic corrections have been determined for entropy and shear viscosity [8]. Reassuringly, they come with the expected sign to connect to perturbative results. ${ }^{1}$ For $N_{c}=3$ and $\alpha_{s}=0.5$, their magnitude is $5 \%$ and $37 \%$ for entropy and shear viscosity, respectively, which (optimistically) gives an idea of the quantitative predictiveness to expect in top-down holographic calculations. Indeed, as will be shown below, the Sakai-Sugimoto model does not only reproduce many qualitative features of QCD but is often quantitatively close. This makes it interesting to apply it to new questions that are presently out of reach of a first-principles approach such as lattice QCD. After a brief review of the Witten-Sakai-Sugimoto model and how it compares quantitatively with real QCD, we will discuss some new results obtained for the spectrum and decay rates of glueballs, and for the phase diagram of QCD in the presence of strong magnetic fields (for the latter see also the recent reviews [10]).

\section{Brief review of the Witten-Sakai-Sugimoto model}

\subsection{Large $N_{c}$ gauge theories and the $\mathrm{AdS}_{5} / \mathrm{CFT}_{4}$ correspondence}

The dominant Feynman diagrams of nonabelian gauge theories in the limit of large number of colors but fixed 't Hooft coupling $\lambda=g^{2} N_{c}$ are the planar diagrams, which can be drawn on a plane (or, equivalently, a sphere) without crossing of lines when the color flow is represented by one line per fundamental or antifundamental index (so that gluons are represented by double lines). Nonplanar diagrams are suppressed by a factor $\left(1 / N_{c}\right)^{\chi}$, where $\chi$ is the Euler number of the surface needed to draw the Feynman diagram. ${ }^{2}$ This is very similar to string perturbation theory, where one has to sum over Riemann surfaces weighted by $g_{s}^{\chi}$ with $g_{s}$ the string coupling constant, leading 't Hooft to the remarkable speculation that the large- $N_{c}$ limit of nonabelian gauge theories may be essentially a string theory [12].

A quarter of a century later, this idea became finally concrete in the form of the AdS/CFT correspondence. The study of nonperturbative objects in string theory, so-called D-branes, led Maldacena

\footnotetext{
${ }^{1}$ In the case of the entropy of a supersymmetric plasma, it is has even been found that the next-to-leading results at weak and at strong coupling can be interpolated uniquely with a singularity-free Padé approximant [9].

${ }^{2} \mathrm{~A}$ nice illustration is provided by Fig. 2 of Ref. [11].
} 
[13] to the conjecture that string theory in the near-horizon limit of $N_{c}$ coincident D3-branes, which is governed by a curved ten-dimensional space of the form $\operatorname{AdS}_{5} \times S^{5}$, is completely equivalent to the low-energy effective theory of these branes in flat Minkowski space, which is the maximally supersymmetric conformal $\mathrm{SU}\left(N_{c}\right)$ gauge theory. Superstring theory, which necessarily involves gravity, in the background of 5-dimensional anti-de Sitter space times a 5-sphere of equal curvature radius is thus proclaimed to give an alternative description of a nonabelian quantum gauge theory in 4 spacetime dimensions without gravity. The latter can be viewed to live on the 4-dimensional (conformal) boundary of anti-de Sitter space, which thus also realizes the holographic principle anticipated for quantum gravity by 't Hooft and Susskind [14].

Spatially three-dimensional branes are nonperturbative objects in type-IIB superstring theory as well as in its low-energy limit, type-IIB supergravity. The latter is a justified approximation when the string length scale $\ell_{s}=\sqrt{\alpha^{\prime}}$ is small compared to spacetime curvature radius ${ }^{3} R$ and when the string coupling $g_{s}$ is small. Because the weak-coupling and strong-coupling description of D3 branes give, respectively, rise to the two relations

$$
g^{2}=4 \pi g_{s}, \quad 4 \pi g_{s} N_{c}=\frac{R^{4}}{\ell_{s}^{4}},
$$

a useful gauge/gravity duality emerges in the limit $g^{2} \rightarrow 0$ and $\lambda \gg 1$, implying $N_{c} \rightarrow \infty$. Useful, because it allows one to substitute extremely difficult calculations in the strongly coupled quantum gauge theory by calculations in weakly-coupled, classical supergravity. (It should be noted, however, that the AdS/CFT correspondence is at its roots a duality between two quantum theories - superstring theory in a certain background on the one hand, and a superconformal field theory on the other.)

The gauge theory in question is however a (maximally) supersymmetric and conformal quantum field theory which is very different from QCD, which is neither supersymmetric nor conformal, but has a running coupling constant and confinement. However, for deconfined QCD at high temperature, the AdS/CFT correspondence may actually be useful. The super-Yang-Mills theory can be considered at finite temperature, where the geometry on the supergravity side becomes one that involves a black hole (strictly speaking a black brane) in anti-de Sitter space. Finite temperature breaks supersymmetry because bosons and fermions have different distribution functions, whereas conformal symmetry may actually be a reasonable approximation sufficiently above the deconfinement temperature. Indeed, the quark-gluon plasma created in heavy-ion collisions seems to behave in many respects like a strongly coupled quantum fluid. In particular hydrodynamic transport coefficients such as shear viscosity turn out to be better described by the famous result $[15,16] \eta / s=\hbar / 4 \pi$ obtained through the AdS/CFT correspondence than by perturbative QCD, where $\eta / s$ is parametrically large. Perturbative QCD is certainly relevant for high-energy processes, and it seems most promising to combine perturbative and strong-coupling gauge/gravity approaches when both have a role to play such as in high-energy jets that are quenched by a strongly coupled medium $[17,18]$.

\subsection{Towards a gravity dual of QCD: the Witten model}

At low temperature, with and without quark chemical potential, superconformal Yang-Mills theory is however certainly very different from real QCD. An intriguing route towards a gravity dual of QCD has been proposed by Witten in Ref. [5]: There are two more fundamental instances of AdS/CFT dualities, arising from the so-called M2- and M5-branes of M-theory, whose low-energy limit is the (unique) 11-dimensional supergravity, already discussed by Maldacena in Ref. [13]. The near-horizon

\footnotetext{
${ }^{3}$ Not to be confused with the Ricci scalar, which will be written as $R^{(d)}$ for a $d$-dimensional space.
} 
geometries of these branes are $\mathrm{AdS}_{4} \times S^{7}$ and $\mathrm{AdS}_{7} \times S^{4}$, respectively, which were found as solutions of 11-dimensional supergravity already in 1980 by Freund and Rubin [19] when they were searching for Kaluza-Klein reductions to 4 spacetime directions. ${ }^{4}$ The dual of M-theory on $\mathrm{AdS}_{7} \times S^{4}$ is the 6-dimensional superconformal low-energy theory of M5 branes. M-theory compactified on a supersymmetry-preserving circle leads to 10-dimensional superstrings of type IIA, with the M5 branes turned into D4 branes. This leads to a nonconformal duality of a 5-dimensional supersymmetric field theory to a string theory on a background that is related to $\mathrm{AdS}_{6} \times S^{4}$ by a Weyl transformation [20]. Witten [5] suggested to consider one further dimensional reduction on a circle for the superfluous fourth spatial coordinate,

$$
x_{4}+2 \pi R_{4} \equiv x_{4}+2 \pi M_{\mathrm{KK}}^{-1} \simeq x_{4},
$$

but with supersymmetry breaking boundary conditions for fermions as they also appear in finite temperature field theory in the imaginary-time formalism. All modes not protected by gauge symmetry become massive in this case, fermionic gluinos at tree-level, and adjoint scalars through quantum effects, leaving pure-glue Yang-Mills theory as low-energy effective theory. The 5-dimensional supersymmetric field theory thus turns into ordinary 4-dimensional Yang-Mills theory. On the other hand, the background geometry of the string theory becomes

$$
d s^{2}=\left(\frac{u}{R}\right)^{3 / 2}\left[\eta_{\mu \nu} d x^{\mu} d x^{v}+f(u) d x_{4}^{2}\right]+\left(\frac{R}{u}\right)^{3 / 2}\left[\frac{d u^{2}}{f(u)}+u^{2} d \Omega_{4}^{2}\right]
$$

with

$$
f(u)=1-\frac{u_{\mathrm{KK}}^{3}}{u^{3}}, \quad M_{\mathrm{KK}}=\frac{3}{2} \frac{u_{\mathrm{KK}}^{1 / 2}}{R^{3 / 2}}
$$

and a nonconstant ("linear" $)$ ) dilaton $e^{\phi}=g_{s}(u / R)^{3 / 4}$. Here $u \geq u_{\mathrm{KK}}$ is the holographic direction with $u=\infty$ corresponding to the conformal boundary. The topology of the subspace formed by $u$ and the Kaluza-Klein direction $x_{4}$ is that of a cigar with the $x_{4}$ circle shrinking to zero size at $u=u_{\mathrm{KK}}$. This lower bound on $u$ corresponds to the would-be horizon of a Euclidean black-hole geometry and the relation between $u_{\mathrm{KK}}$ and $M_{\mathrm{KK}}$ is determined by the absence of a conical singularity at $u=u_{\mathrm{KK}}$.

The parameters of the 4-dimensional gauge theory with Lagrangian $\mathcal{L}=-\frac{1}{2 g^{2}} \operatorname{Tr} F_{\mu \nu} F^{\mu \nu}$ resulting from the reduction of $\mathcal{L}=-\frac{1}{2 g_{5}^{2}} \operatorname{Tr} F_{M N} F^{M N}+\ldots$ with $g_{5}^{2}=2 g_{s}(2 \pi)^{2} \ell_{s}$ are given by ${ }^{6}$

$$
g^{2}=\frac{g_{5}^{2}}{2 \pi R_{4}}=4 \pi g_{s} \ell_{s} M_{\mathrm{KK}}, \quad \frac{R^{3}}{\ell_{s}^{3}}=\pi g_{s} N_{c},
$$

where the latter equation is due to the nonvanishing Ramond tensor field sourced by the $N_{c}$ D4-branes. For the supergravity approximation to be justified, the maximum curvature $\sim\left(u_{\mathrm{KK}} R^{3}\right)^{-1 / 2}$ located at $u=u_{\mathrm{KK}}$ needs to be small compared to the string tension $\propto \ell_{s}^{-2}$, which again requires large 't Hooft coupling $\lambda=g^{2} N_{c} \gg 1$.

The dimensionless coupling $g$ is derived from the dimensionful coupling $g_{5}$ at the Kaluza-Klein mass scale $M_{\mathrm{KK}}$, beyond which the field theory reveals its underlying 5-dimensional nature. Ideally,

\footnotetext{
${ }^{4}$ These solutions are stabilized by having constant flux from the antisymmetric tensor fields of 11-dimensional supergravity on the compact spheres, for which the M-branes provide sources. Because the anti-de Sitter space and the sphere have comparable curvature, these solutions are not viable as Kaluza-Klein theories for the real world, but they paved the way to more elaborate flux compactifactions in string theory.

${ }^{5}$ Since it would be linear in suitable radial coordinates.

${ }^{6}$ In virtually all of the literature on the Witten(-Sakai-Sugimoto) model, $\mathcal{L}=-\left(4 g_{\mathrm{YM}}^{2}\right)^{-1} \operatorname{Tr} F_{\mu \nu} F^{\mu v}$ is used. This different convention has no consequences as long as no comparison to perturbative QCD is made, where the standard definition of the $\mathrm{SU}(3)$ gauge coupling is $g$ with $g^{2}=2 g_{Y M}^{2}$ and the $\alpha_{s}$ of QCD reads $\alpha_{s}=g^{2} / 4 \pi=g_{Y M}^{2} / 2 \pi$.
} 


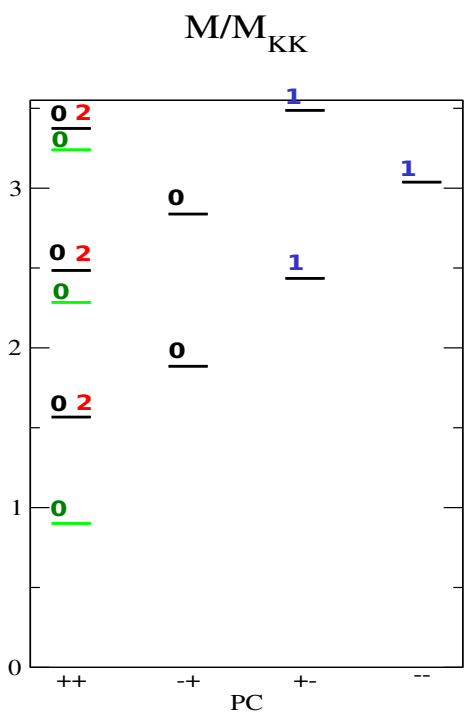

(a)

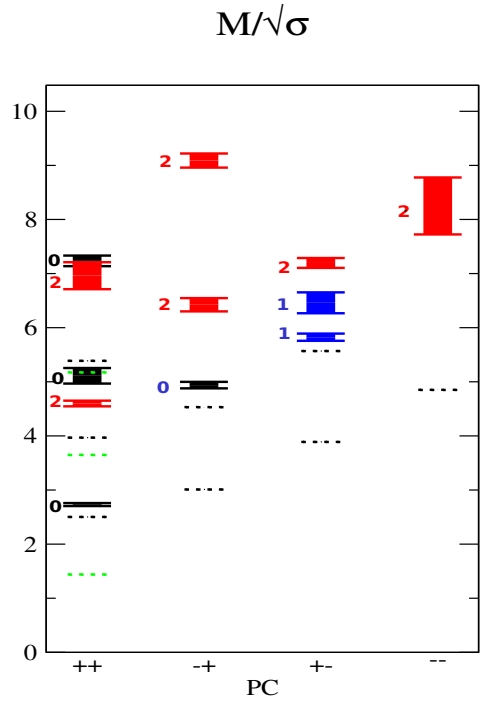

(b)

Figure 1. Glueball spectrum of the Witten model (a) as obtained in Ref. [21], in units of $M_{\mathrm{KK}}$, ("exotic" modes in green), compared to the recent large- $N_{c}$ lattice results of Ref. [22] (b) in units of $\sqrt{\sigma}$ but with overall scale such that the lowest tensor mode are on equal height. The dotted lines in (b) give the holographic spectrum in terms of the holographic string tension with the standard parameter set of the Sakai-Sugimoto model (15).

$M_{\mathrm{KK}}$ should be sent to infinity, but that would mean small 't Hooft coupling, where string theory effects beyond the supergravity approximation would be needed. In the Witten model, we have to be content with the strong-coupling regime below some finite scale $M_{\mathrm{KK}}$, but the fact that this dual theory may be continuously connected to real QCD makes it an interesting approximation to the strong-coupling regime of the latter.

A particularly simple quantity to calculate in the Witten model is the Wilson loop of heavy (nondynamical) quarks. Connecting two heavy quarks at the boundary which are widely separated along $x$ through a Wilson line is represented by a fundamental string embedded in the bulk geometry. The string tension is locally given by $\left(2 \pi \alpha^{\prime}\right)^{-1} \sqrt{-g_{t t} g_{x x}}$. For large separation in $x$, the energetically favored solution is clearly to have as much as possible of the length of the string at the minimal value of $\sqrt{-g_{t t} g_{x x}}$, which occurs at $u=u_{\mathrm{KK}}$. The effective string tension at large $\Delta x$ thus approaches a constant which indicates confinement of quarks and which is given explicitly by

$$
\sigma=\left.\frac{1}{2 \pi \ell_{s}^{2}} \sqrt{-g_{t t} g_{x x}}\right|_{u=u_{\mathrm{KK}}}=\frac{1}{2 \pi \ell_{s}^{2}}\left(\frac{u_{\mathrm{KK}}}{R}\right)^{3 / 2}=\frac{g^{2} N_{c}}{27 \pi} M_{\mathrm{KK}}^{2} .
$$

In accordance with confinement, there is a mass gap in the spectrum of fluctuations of the background geometry which is proportional to $M_{\mathrm{KK}}$. These fluctuations can be classified with respect to their 4-dimensional quantum numbers and comprise scalar, vector, and tensor modes, which correspond to glueballs in the gauge theory.

Following up on pioneering work in Ref. [23-25], the complete spectrum was worked out in Ref. [21]. In Fig. 1, the spectrum in the Witten model (Fig. 1a) is compared with recent lattice results [22] for large- $N_{c}$ Yang-Mills theory (colored boxes in Fig. 1b) with the vertical scale chosen such that the lowest tensor glueball $2^{++}$in the two figures have equal height. The holographic calculation 
appears to reproduce qualitatively many of the conspicuous features of the lattice result which is in fact rather similar to those obtained at $N_{c}=3$. The main qualitative difference is the absence of glueballs with quantum numbers $2^{-+}$and of glueballs with spin $>2$ in the Witten model. Another peculiar feature of the holographic result is a certain proliferation of scalar modes $0^{++}$: the green lines in Fig. 1a represent scalar modes which have a polarization involving the metric component $g_{44}$ and which have been termed "exotic" in Ref. [25]. The other modes in the $P C=++$ sector are degenerate $0^{++}$and $2^{++}$glueballs. Thus there are 4 scalar glueballs with mass less or equal the first excited tensor glueballs, whereas the lattice features only two in that range. In fact, Ref. [25] already suspected that the scalar glueballs associated with the "exotic" polarization may perhaps not correspond to glueballs in QCD, but later Ref. [21] found that the lowest exotic mode is the lowest-lying one of all the modes, making the overall picture tantalizingly similar to that found in lattice simulations. Below we shall revisit this issue when quarks are included by the extension provided by the Witten-Sakai-Sugimoto model.

In Ref. [20] the necessary tools for holographic renormalization for nonconformal holography have been developed and applied to the Witten model to calculate the gluon condensate with the result

$$
\frac{1}{4}\left\langle\operatorname{Tr} F^{2}\right\rangle=\frac{2 N_{c}}{3^{7} \pi^{2}}\left(g^{2} N_{c} / 2\right)^{2} M_{\mathrm{KK}}^{4},
$$

where we have taken into account that the $g_{4}$ used in Ref. [20] differs from the standard definition of $g$ of perturbative QCD by $g_{4}^{2}=g^{2} / 2$. The gluon condensate as usually defined in QCD in terms of canonically normalized gluon fields $G_{\mu \nu}^{a}$ is given by

$$
C^{4} \equiv\left\langle\frac{\alpha_{s}}{\pi} G_{\mu \nu}^{a} G^{a \mu \nu}\right\rangle=\frac{1}{2 \pi^{2}}\left\langle\operatorname{Tr} F^{2}\right\rangle=\frac{4 N_{c}}{3^{7} \pi^{4}}\left(g^{2} N_{c} / 2\right)^{2} M_{\mathrm{KK}}^{4} .
$$

We shall come back to this when discussing the choice of parameters of the Sakai-Sugimoto model.

\subsection{Inclusion of chiral quarks: the Sakai-Sugimoto model}

Type-IIA string theory has stable D-branes with any even number of spatial dimensions. If such additional branes are present, the open strings connecting those with the color D4-branes carry one color index on one end and a new index on the other, which can represent the flavor quantum number of quarks. If flavor and color branes intersect, one gets chiral quarks. As long as the number $N_{f}$ of these additional branes is small compared to $N_{c}$, they can be considered as "probes" with negligible back-reaction on the background geometry they are imbedded in. This is analogous to the introduction of quenched quarks in lattice QCD.

A first interesting attempt to include quarks in the Witten model was made by Kruczenski, Mateos, Myers, and Winters [26] by introducing D6 flavor branes in the background of the Witten model. In the case of only one flavor brane and a massless quark, a spontaneous breaking of an axial U(1) symmetry was observed, but the generalization to a nonabelian flavor symmetry at $N_{f}>1$ did not give a good model for chiral symmetry breaking.

This problem was solved by Sakai and Sugimoto [6, 7] by instead using pairs of probe ${ }^{7}$ D8 and anti-D8 branes filling all spatial directions except the $x_{4}$ (Fig. 2a). This introduces chiral quarks and anti-quarks localized at different points of the Kaluza-Klein circle. The global flavor symmetry $\mathrm{U}\left(N_{f}\right)_{L} \times \mathrm{U}\left(N_{f}\right)_{R}$ of the field theory living on the boundary corresponds to a local gauge symmetry on the flavor branes, which is broken spontaneously to the diagonal subgroup because the D8 and anti-D8 branes have nowhere to end except by joining in the bulk (Fig. 2b).

\footnotetext{
${ }^{7}$ The daunting issue of backreaction of localized D8 branes has been studied in Ref. [27]. A different strategy to include dynamical quarks has been recently proposed in Ref. [28] through smeared D8 branes and corrections to first order in $N_{f} / N_{c}$ have been calculated. A price of the latter approach is however that the global flavor symmetry is reduced to $\mathrm{U}(1)_{L}^{N_{f}} \times \mathrm{U}(1)_{R}^{N_{f}}$.
} 


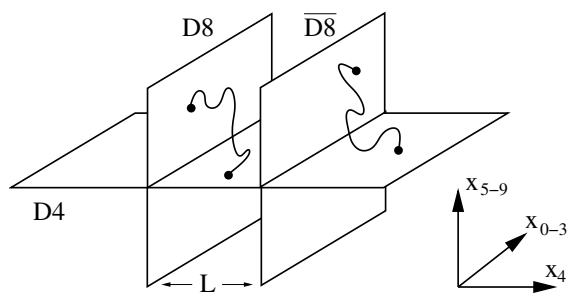

(a)

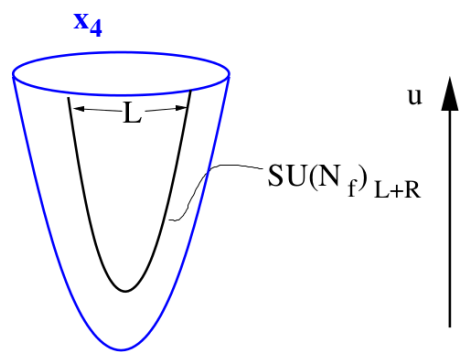

(b)

Figure 2. (a) D4-D8- $\overline{\mathrm{D} 8}$ brane configuration in the 10 dimensions $x_{0}, \ldots, x_{9}$, with $x_{4}$ understood as periodic; (b) near-horizon geometry with $u$ being a radial coordinate in the transverse space $x_{5 \ldots .}$.

The spectrum of fluctuations of the flavor gauge fields on the D8 branes include a zero mode for the fifth component $A_{u}\left(x^{\mu}, u\right)$ whose gauge-invariant holonomy represents the Goldstone bosons of the spontaneously broken $\mathrm{U}_{A}\left(N_{f}\right)$ :

$$
U\left(x^{\mu}\right)=\mathrm{P} \exp \left\{i \int_{-\infty}^{\infty} d Z^{\prime} A_{Z}\left(x^{\mu}, Z\right)\right\} \equiv \exp \left\{2 i \pi\left(x^{\mu}\right) / f_{\pi}\right\} \in \mathrm{U}\left(N_{f}\right),
$$

where we have switched to a new dimensionless coordinate $Z$ defined by $\left(u / u_{\mathrm{KK}}\right)^{3}=1+Z^{2}$ which runs from $-\infty$ to $+\infty$ as one follows the holographic direction from the boundary into the bulk along the D8 brane and back along the anti-D8 brane. Here we have assumed that the D8 and the anti-D8 brane are maximally separated so that they connect at the tip of the cigar, $u=u_{\mathrm{KK}}$.

In massless QCD, the axial $\mathrm{U}(1)_{A}$ symmetry is broken by an anomaly, which is suppressed at large $N_{c}$. However, this effect has also a beautiful supergravity description [29] involving the RamondRamond 1-form whose flux through the surface parametrized by $x_{4}$ and $u$ is related to the $\theta$ parameter. The mass of the $\eta^{\prime}$ pseudoscalar meson is related by the Witten-Veneziano formula to the topological susceptibility, and was calculated by Sakai and Sugimoto [6] as ${ }^{8}$

$$
m_{\eta^{\prime}}=\frac{1}{3 \sqrt{3} \pi} \sqrt{\frac{N_{f}}{N_{c}}}\left(g^{2} N_{c} / 2\right) M_{\mathrm{KK}}
$$

To leading order in $N_{c}$ and $\alpha^{\prime}$, the D8-brane action is given explicitly by

$$
S_{\mathrm{D} 8}=-\kappa \int d^{4} x d Z \operatorname{Tr}\left[\frac{1}{2}\left(1+Z^{2}\right)^{-1 / 3} F_{\mu \nu}^{2}+\left(1+Z^{2}\right) M_{\mathrm{KK}}^{2} F_{\mu Z}^{2}+O\left(\alpha^{\prime 2} F^{4}\right)\right]+S_{\mathrm{CS}}, \quad \kappa=\frac{\left(g^{2} N_{c} / 2\right) N_{c}}{216 \pi^{3}},
$$

where all fields have been taken as constant with respect to the $S^{4}$ whose volume has been integrated over. $S_{\mathrm{CS}}$ is the unavoidable Chern-Simons action of D-branes which here can be shown to reproduce the correct chiral anomaly of QCD, and also the correct Wess-Zumino-Witten term in the chiral Lagrangian [6]. The well-known 5-dimensional formulation of the latter thus appears in a most natural way; background gauge fields for the chiral symmetry can be introduced as nontrivial boundary conditions $A_{\mu}^{L, R}(x)=\lim _{Z \rightarrow \pm \infty} A_{\mu}(Z, x)$.

\footnotetext{
${ }^{8}$ Note that Ref. [6] uses $g_{\mathrm{YM}}$ and that our $g^{2}=2 g_{\mathrm{YM}}^{2}$.
} 
The Goldstone bosons and the $\eta^{\prime}$ meson are contained in $A_{Z}=\phi_{0}(Z) \pi(x)$ with $\phi_{0} \propto\left(1+Z^{2}\right)^{-1}$. Requiring a canonical normalization of the kinetic term for the Goldstone bosons in

$$
S_{\mathrm{D} 8}=\frac{f_{\pi}^{2}}{4} \int d^{4} x \operatorname{Tr}\left(U^{-1} \partial_{\mu} U\right)^{2}+\ldots,
$$

fixes the so-called pion decay constant in terms of $\lambda$ and $M_{\mathrm{KK}}$ as

$$
f_{\pi}^{2}=\frac{1}{54 \pi^{4}}\left(g^{2} N_{c} / 2\right) N_{c} M_{\mathrm{KK}}^{2}
$$

Vector and axial vector mesons appear as even and odd eigenmodes of $A_{\mu}^{(n)}=\psi_{n}(Z) v_{\mu}^{(n)}(x)$ with equation

$$
-\left(1+Z^{2}\right)^{1 / 3} \partial_{Z}\left(\left(1+Z^{2}\right) \partial_{Z} \psi_{n}\right)=\lambda_{n} \psi_{n}, \quad \psi_{n}( \pm \infty)=0 .
$$

The lowest mode is interpreted as the vector meson $\rho$ with mass $m_{\rho}^{2}=\lambda_{1} M_{\mathrm{KK}}^{2}$ and $\lambda_{1}=0.669$. The next mode with $\lambda_{2}=1.569$ is an axial vector meson that can be identified [6] with the $a_{1}(1260)$. The experimental value $m_{a_{1}(1260)}^{2} / m_{\rho}^{2} \approx 2.52$ is surprisingly close to $\lambda_{2} / \lambda_{1} \approx 2.344$. Also the ratio of the squared mass of the next vector meson, $\rho(1450)$, to that of the $\rho$ meson, $m_{\rho(1450)}^{2} / m_{\rho}^{2} \approx 3.57$ compares quite well with $\lambda_{3} / \lambda_{1} \approx 4.29$. However, this nice agreement may be fortuitous. Recent lattice simulations of the spectrum of mesons in large- $N_{c}$ QCD extrapolated to zero quark masses have obtained larger values [30]: $m_{a_{1}}^{2} / m_{\rho}^{2} \approx 3.46$ and $m_{\rho^{*}}^{2} / m_{\rho}^{2} \approx 5.77$. This would corresond to an error of $21 \%$ and $16 \%$, respectively, for the mass ratios (unsquared). Given that the Sakai-Sugimoto model is bound to show deviations from QCD above the scale of $M_{\mathrm{KK}} \approx 1.22 m_{\rho}$, this may still be taken as a notable success.

In Ref. [6, 7] the physical values of $m_{\rho} \approx 776 \mathrm{MeV}$ and $f_{\pi} \approx 92.4 \mathrm{MeV}$ have been used to set the free parameters

$$
M_{\mathrm{KK}}=949 \mathrm{MeV}, \quad \kappa=7.45 \cdot 10^{-3} \Rightarrow \bar{\lambda} \equiv g^{2} N_{c} / 2 \approx 16.63,
$$

$\left(\bar{\lambda}=g_{\mathrm{YM}}^{2} N_{c}\right.$ is the 't Hooft coupling as used in Ref. [6, 7]; cf. footnote 6), and these values have been used widely since. At $N_{c}=3$, this amounts to $\alpha_{s}\left(M_{\mathrm{KK}}\right) \approx 0.88$, which is of the order of magnitude to expect from perturbative QCD at such low scales, albeit somewhat high when compared to the particular values obtained in the $\overline{\mathrm{MS}}$-scheme, $\bar{\alpha}_{s}(1 \mathrm{GeV}) \approx 0.5$. But one should keep in mind that $\bar{\alpha}_{s}$ grows sharply at $1 \mathrm{GeV}$ with large renormalization scheme dependence. A better quantity to consider is the renormalization-scheme independent string tension. The holographic result (6) depends on both $M_{\mathrm{KK}}$ and $\bar{\lambda}$, which yields with the above choice (15) $\sqrt{\sigma}=0.6262 M_{\mathrm{KK}} \approx 594 \mathrm{MeV}$. This is higher than the value $\sqrt{\sigma} \sim 440 \mathrm{MeV}$ extracted from lattice calculations [31], also suggesting that $\bar{\alpha}_{s}$ is somewhat too large, since in (6) $\sigma \propto \alpha_{s}$.

Another quantity of immediate interest which depends on both $M_{\mathrm{KK}}$ and $\bar{\lambda}$ is the gluon condensate. (The glueball spectrum, which only depends on $M_{\mathrm{KK}}$, will be revisited in the next section.) Inserting the parameters (15) and $N_{c}=3$ yields

$$
C^{4}=0.0126 \mathrm{GeV}^{4} \quad(\bar{\lambda}=16.63)
$$

which happens to almost coincide with the standard SVZ sum rule [32] value $C^{4}=0.012 \mathrm{GeV}^{4}$ in QCD. However, both significantly smaller [33, 34] and larger values [35] have been obtained using sum rules. Lattice simulations typically give significantly larger values, but of the same size as ambiguities from the subtraction procedure [36]. Thus it appears that the gluon condensate is not very 
suited to really determine the 't Hooft coupling in the Witten(-Sakai-Sugimoto) model. A moderate value of the gluon condensate may however be considered to be a prediction of this model.

Using the result for the string tension, one can also compare the prediction for the dimensionless ratio

$$
m_{\rho} / \sqrt{\sigma} \approx 1.306 \quad(\bar{\lambda}=16.63)
$$

with the large- $N_{c}$ lattice result of Ref. [30], which reads 1.504(50). This agrees within $15 \%$, and the fact that the holographic result is smaller also suggests that the assumed value for $\bar{\lambda}$ and thus $\sigma$ in the Sakai-Sugimoto model is perhaps too large. Turning this around, and fitting the lattice result for $m_{\rho} / \sqrt{\sigma}$ would give $\bar{\lambda} \approx 12.55(80)$ resulting in more moderate values $\sqrt{\sigma} \approx 515 \mathrm{MeV}$ and $\alpha_{s}\left(M_{\mathrm{KK}}\right) \approx$ 0.66 . This also corresponds to a $13 \%$ lower value of the pion decay constant, $f_{\pi}=80.3 \mathrm{MeV}$, and one might indeed argue in favor of a reduced $f_{\pi}$ because the Sakai-Sugimoto model is strictly chiral (although, judging from the lattice results of Ref. [37], $f_{\pi}$ should have a reduction around $7 \%$ for $N_{c}=3$, whereas at large $N_{c}$ Ref. [30] found only a 2\% reduction). At any rate, a downward variation of $\bar{\lambda}$ from 16.63 , say to 12.55 , should give a reasonable theoretical error bar for quantitative predictions of the Witten-Sakai-Sugimoto model.

In Ref. [38], Imoto, Sakai, and Sugimoto have more recently studied the predictions of the SakaiSugimoto model for other mesons, which are not covered by the flavor gauge fields on the D8-branes. The latter correspond to massless open string modes and only give the Goldstone bosons and a tower of $1^{--}$and $1^{++}$mesons. ${ }^{9}$ Despite the fact that gauge/gravity duality works in the decoupling limit corresponding to $\ell_{s} \rightarrow 0$, the masses of the massive string modes remain finite, and yield a host of mesonic states. The spectrum of those further states is very difficult to evaluate precisely because of the curved background. Ref. [38] succeeded, however, in calculating corrections to the Regge trajectory of the $\rho$-meson and could reproduce the required curvature of the trajectory around zero mass to match with a Regge intercept $\alpha_{0}=1$. In order to match the experimental values of the meson masses on the $\rho$-trajectory, Ref. [38] again observed that the model needs a lower value of the string tension than given by the standard choice of parameters. Their fit even points to a rather low value of $\sqrt{\sigma}=380 \mathrm{MeV}$. Curiously enough, also the large- $N_{c}$ lattice study of Ref. [30] found a reduced value of $\sqrt{\sigma}$, in their case $395 \mathrm{MeV}$, to give a better fit than $440 \mathrm{MeV}$.

The Sakai-Sugimoto model is not limited to the meson sector of low-energy QCD. The D8-brane action (12), restricted to the Goldstone bosons and integrated over the holographic coordinate, reads, to leading order in 't Hooft coupling and $N_{c}$ :

$$
S_{\mathrm{D} 8}=\int d^{4} x\left\{\frac{f_{\pi}^{2}}{4} \operatorname{Tr}\left(U^{-1} \partial_{\mu} U\right)^{2}+\frac{1}{32 e^{2}} \operatorname{Tr}\left[U^{-1} \partial_{\mu} U, U^{-1} \partial_{\nu} U\right]^{2}\right\}, \quad e^{-2}=\frac{15.253}{27 \pi^{7}} g^{2} N_{c}^{2},
$$

with $f_{\pi}^{2} \propto g^{2} N_{c}^{2}$ given already in (12). This coincides with the Skyrme model [39] with a definite prediction of the Skyrme parameter $e$ and so allows baryons to appear as solitons of the chiral Lagrangian. In the bulk, the corresponding object is a holographic baryon vertex [40] in the form of additional D4-branes wrapping the $S^{4}$ in the 10-dimensional background geometry. Because of the RR flux on the $S^{4}$ the D4-brane is connected to the D8 branes by $N_{c}$ open strings, which pull it inside the D8 branes where it appears as an instanton configuration, realizing a connection between instantons in 5-dimensional gauge theory and the Skyrmion recognized prior to the Sakai-Sugimoto model in Ref. [41].

\footnotetext{
${ }^{9}$ Originally, also excitations of the brane embedding function were considered in Ref. [6] and interpreted as a tower of $0^{++}$ and $0^{--}$scalar mesons. These are, however, odd under the so-called [21] $\tau$-parity, and should be discarded since the quarks and gluon are invariant under this discrete symmetry [38]. It is therefore not disturbing that the prediction for the mass of the $a_{0}(1450)$ meson in the original paper of Sakai and Sugimoto does not agree well with the recent large- $N_{c}$ lattice result of Ref. [30].
} 
A full-fledged treatment of baryons in the Sakai-Sugimoto model has proved to be very interesting but difficult [42-45], suggesting that string corrections should be taken into account. Considering the baryon mass, it should be given to leading order by the mass of a D4-brane at the tip of the D8 brane configuration, which is [6] $m_{\mathrm{D} 4}=8 \pi^{2} \kappa M_{\mathrm{KK}}=\frac{1}{27 \pi}\left(g^{2} N_{c} / 2\right) N_{c} M_{\mathrm{KK}}$. In [42, 43] the correction coming from the $\mathrm{U}(1)_{V}$-field induced on the D8-brane and leading to a contribution from $S_{\mathrm{CS}}$ has been calculated with the result $[42,43]$

$$
M_{B}=\left[\frac{1}{27 \pi}\left(g^{2} N_{c} / 2\right)+\sqrt{\frac{2}{15}}+O\left(\left(g^{2} N\right)^{-1}\right)\right] N_{c} M_{\mathrm{KK}} .
$$

With the standard parameter choice this corresponds to $(558+1040=1598) \mathrm{MeV}$, which is too high when compared to real nucleons. But the lack of convergence (the correction term is about twice the leading term) seems to indicate that an expansion about a pointlike instanton is problematic, at least for the 't Hooft coupling required to make contact with QCD. Curiously enough, if one just considers the effective Skyrme action (18) and evaluates the well-know result for the energy of a Skyrmion $[39,46], E \approx 23.2 \pi f_{\pi} / e$, with the above parameters, one obtains $M_{N} \approx 920 \mathrm{MeV}$, tantalizingly close to the real-world value. This may be taken as an indication that one need not lower the scale $M_{\mathrm{KK}}$ to make contact with QCD (as suggested in [43]), but should rather aim at a different approximation scheme for holographic baryons.

As another extreme we can also consider the mass of the $\eta^{\prime}$-meson given in (10), which is proportional to $\sqrt{N_{f} / N_{c}}$ and thus parametrically suppressed at large $N_{c}$. However, extrapolating to $N_{c}=3$ and inserting again the above parameters for $\bar{\lambda}$ and $M_{\mathrm{KK}}$ this yields $m_{\eta^{\prime}} \approx 967 \mathrm{MeV}$ for $N_{f}=3$, astonishingly close to the experimental value of $958 \mathrm{MeV}$. With the alternative choice $\bar{\lambda}=12.55$, the lower value of $730 \mathrm{MeV}$ is obtained, which is seemingly disfavored, but may actually be more appropriate because of the chiral limit and the missing strange quark mass.

In Table 1, a summary of the numerical predictions are given, including for completeness a variation of the Sakai-Sugimoto model where the D8 branes are not maximally separated [47, 48]. They then join at a point $u_{0}>u_{\mathrm{KK}}$, and one may interpret the difference between $u_{0}$ and $u_{\mathrm{KK}}$ as a constituent quark mass, given by the mass of a string stretched from $u_{0}$ ot $u_{\mathrm{KK}}$. In Ref. [49] the latter has been set to $310 \mathrm{MeV}$, which after matching the $\rho$ meson mass leads to a significantly smaller value of $M_{\mathrm{KK}}$ and a slighter smaller value of $\bar{\lambda}$. As remarked in Ref. [49], this leads to a better match of the string tension as observed on the lattice. On the other hand, the gluon condensate becomes uncomfortably small. Also the other meson masses fit somewhat less well.

To wrap up this numerological discussion, the original choice of parameters of the Sakai-Sugimoto model seems to be in quite good shape, but one may want to consider also smaller 't Hooft couplings in view of the somewhat high string tension. At any rate, the fact that most of the quantities considered here come out in the right ballpark is certainly remarkable given that this model has a cutoff scale of $M_{\mathrm{KK}} \sim 1 \mathrm{GeV}$. A reason may be that this cutoff is very smooth in comparison with a sharp momentum or lattice cutoff and that it also fully respects 3+1-dimensional Lorentz symmetry.

\section{Glueball spectrum and glueball decay rates}

With the above successes as motivation, we now come back to the glueball spectrum as obtained already in 2000 in Ref. [21] and displayed in Fig. 1a. Here the scale is determined by $M_{\mathrm{KK}}$ alone. With $M_{\mathrm{KK}}=949 \mathrm{MeV}$, the lowest scalar mode (lowest green line in Fig. 1a) turns out ot have a mass of $M_{G} \approx 855 \mathrm{MeV}$, only marginally heavier than the $\rho$ meson. Lattice simulations in QCD give values around $1.7 \mathrm{GeV}$, a factor of 2 higher. This mismatch also persists when one compares with the large- $N_{c}$ lattice results of [22]: $M_{G} / \sqrt{\sigma}$ is $\approx 1.44$ with the parameters of the Sakai-Sugimoto 
Table 1. Choice of parameters of the Witten-Sakai-Sugimoto model and resulting predictions in 3 variants: (i) Original choice of Sakai and Sugimoto [7] which fits $M_{\mathrm{KK}}$ to the experimental value of $m_{\rho}$ and the 't Hooft coupling $\bar{\lambda}$ through the experimental value of $f_{\pi}$; (ii) same, except that $\bar{\lambda}$ is fitted such as to reproduce the large- $N_{c}$ lattice result of Ref. [30] for $m_{\rho} / \sqrt{\sigma} \approx 1.504$; (iii) non-antipodal D8 brane configuration with $u_{0} / u_{\mathrm{KK}} \approx 1.38$ such as to have a constituent quark mass of $m_{q}^{\text {constit. }}=310 \mathrm{MeV}$ [49]. (Starred numbers are directly fixed by the input parameters.)

\begin{tabular}{l|lll} 
& $\begin{array}{l}\text { (i) antipodal [7] } \\
\left(m_{\rho}, f_{\pi}\right)\end{array}$ & $\begin{array}{l}\text { (ii) antipodal } \\
\left(m_{\rho}, m_{\rho} / \sqrt{\sigma}\right)\end{array}$ & $\begin{array}{l}\text { (iii) non-antipodal [49] } \\
\left(m_{\rho}, f_{\pi}, m_{q}^{\text {constit. }}\right)\end{array}$ \\
fitted to: & 949 & 949 & 720.1 \\
$M_{\mathrm{KK}}[\mathrm{MeV}]$ & 16.63 & 12.55 & 15.13 \\
$\bar{\lambda}\left(=6 \pi \alpha_{s}\right)$ & $92.4^{*}$ & 80.3 & $93^{*}$ \\
\hline$f_{\pi}[\mathrm{MeV}]$ & $776^{*}$ & $776^{*}$ & $776^{*}$ \\
$m_{\rho}[\mathrm{MeV}]$ & 594 & $515^{*}$ & 430 \\
$\sqrt{\sigma}[\mathrm{MeV}]$ & 0.0126 & 0.0072 & 0.0035 \\
$C^{4}\left[\mathrm{GeV}^{4}\right]$ & 967 & 730 & 667 \\
$\left.m_{\eta^{\prime}}\right|_{f_{f}=3}[\mathrm{MeV}]$ & 1188 & 1188 & 1146 \\
$m_{a_{1}(1260)}[\mathrm{MeV}]$ & 1188 & 855 & 649 \\
$M_{G}[\mathrm{MeV}]$ & 855 & 1487 & 1128 \\
$M_{D, T}[\mathrm{MeV}]$ & 1487 & &
\end{tabular}

Table 2. Holographic glueball masses $\left(M_{\mathrm{KK}}=949 \mathrm{MeV}\right)$. The glueball candidate corresponding to the "exotic" polarization of the metric field is marked by "(E)".

\begin{tabular}{l|rr}
$J^{P C}$ & $M / M_{\mathrm{KK}}$ & $M[\mathrm{MeV}]$ \\
\hline $0^{++}(\mathrm{E})$ & 0.90113 & 855 \\
$0^{++}-2^{++}$ & 1.56690 & 1487 \\
$0^{-+}$ & 1.88518 & 1789 \\
$0^{*++}(\mathrm{E})$ & 2.28487 & 2168 \\
$1^{+-}$ & 2.43529 & 2311 \\
$0^{*++} 2^{*++}$ & 2.48514 & 2358 \\
$0^{*-+}$ & 2.83783 & 2693 \\
$1^{--}$ & 3.03763 & 2883 \\
\hline
\end{tabular}

Table 3. The masses of isotriplet mesons given by the modes of the flavor gauge field on the (maximally separated) D8 branes, compared to the experimental value $\left(m / m_{\rho}\right)^{\text {exp. from [PDG] }}$ $\left(\rho(770), a_{1}(1260), \rho(1450), a_{1}(1640)\right)$ and to the large- $N_{c}$ results from Ref. [30].

\begin{tabular}{l|llll} 
Isotriplet Meson & $\lambda_{n}=m^{2} / M_{\mathrm{KK}}^{2}$ & $m / m_{\rho}$ & $\left(m / m_{\rho}\right)^{\text {exp. }}$ & $m / m_{\rho}[30]$ \\
\hline $0^{-+}(\pi)$ & 0 & 0 & $0.174 \mid 0.180$ & 0 \\
$1^{--}(\rho)$ & 0.669314 & 1 & 1 & 1 \\
$1^{++}\left(a_{1}\right)$ & 1.568766 & 1.531 & $1.59(5)$ & $1.86(2)$ \\
$1^{--}\left(\rho^{*}\right)$ & 2.874323 & 2.072 & $1.89(3)$ & $2.40(4)$ \\
$1^{++}\left(a_{1}^{*}\right)$ & 4.546104 & 2.606 & $2.12(3)$ & $2.98(5)$ \\
\hline
\end{tabular}


model, while Ref. [22] finds $\approx 2.73$ for the lowest scalar glueball (see Fig. 1b, where the dotted lines display the holographic results for the glueball spectrum normalized to $\sqrt{\sigma}$ ). The generalization of the Sakai-Sugimoto model, where the D8 branes are not maximally separated, does not help in this respect. On the contrary, it increases $m_{\rho} / M_{\mathrm{KK}}$ while keeping $M_{G} / M_{\mathrm{KK}}$ fixed. (See Table 1: For the choice of parameters of Ref. [49] for this generalized Sakai-Sugimoto model, $M_{G}$ is already below $m_{\rho}$.)

As already discussed, while the glueball spectrum in the Witten model resembles the spectrum as found on the lattice, it also has some notable qualitative differences such as a certain proliferation of scalar glueballs $0^{++}$. Moreover, the lowest holographic glueball comes from an "exotic" $g_{44}$ polarization of the metric fluctuation (while also involving other metric components as well as the dilaton). The next higher scalar glueball, which has the same mass as the lowest tensor glueball, does not involve a $g_{44}$ fluctuation, and could thus be viewed as essentially a dilaton fluctuation, which in simpler bottom-up models [50-52] is the only way to model a scalar glueball. The mass of the latter is $M_{D}=M_{T} \approx 1.567 M_{\mathrm{KK}} \approx 1487 \mathrm{MeV}$, which matches reasonably well with the lowest scalar glueball on the lattice (albeit not for the tensor glueball which on the lattice is around 2.4-2.6 GeV).

An important open question, which is difficult to address by lattice simulations, is the decay width of glueballs. In the Witten model, holographic glueballs are stable modes, and they remain so when one introduces chiral quarks through probe D8 branes. However, as pointed out by Ref. [53], the metric fluctuations dual to glueballs couple to the modes on the D8 brane representing pions and vector mesons. Ref. [53] has considered the decay rate of the lowest ("exotic") scalar mode, which was revisited and extended recently by Brünner, Parganlija and the present author [54, 55].

In order to see whether the Sakai-Sugimoto model has any chance to predict glueball decay rates, let us begin with considering the decay of the $\rho$ meson first. The flavor gauge field modes on the D8 brane are all stable, but the D8-brane action determines all the couplings between different mesons without further free parameters. To leading order they have been worked out completely in Ref. [7], but only a few (particularly interesting and challenging) decay processes have been discussed in the literature.

It is instructive to consider one of the simplest cases, the decay of the $\rho$ meson, to see whether one may hope for quantitatively significant predictions. This was done already in Ref. [53], but unfortunately using parameters affected by an error in the normalization of the D8-brane action present in the original and published version of Ref. [6, 7] for the multi-flavor case $N_{f}>1$ (corrected however in the final e-print version). Identifying the mode $n=1$ in (14) with the $\rho$ meson, $A_{\mu}^{(1)}=\psi_{1}(Z) v_{\mu}^{(1)}(x) \equiv$ $\psi_{1}(Z) \rho_{\mu}(x)$ and ensuring that $\rho(x)$ is a canonically normalized vector field yields

$$
\mathcal{L}_{\rho \pi \pi}=-g_{\rho \pi \pi} \epsilon_{a b c}\left(\partial_{\mu} \pi^{a}\right) \rho^{b \mu} \pi^{c}, \quad g_{\rho \pi \pi}=\sqrt{2} \int d Z \frac{1}{\pi K} \psi_{1}=\sqrt{2} \times 24.030 \bar{\lambda}^{-\frac{1}{2}} N_{c}^{-\frac{1}{2}} .
$$

$\left(g_{\rho \pi \pi} / \sqrt{2}\right.$ was denoted as $c_{6}$ in [53].)

Using the original set of parameters (15) gives a decay rate of the $\rho$ meson in two (massless) pions

$$
\Gamma_{\rho} / m_{\rho}=\frac{g_{\rho \pi \pi}^{2}}{48 \pi} \approx 7.659\left(\bar{\lambda} N_{c}\right)^{-1} \approx 0.1535
$$

which is $20 \%$ lower than the experimental value $\Gamma_{\rho} / m_{\rho}=0.191(1)$ from the PDG [56]. As we have discussed above, the coupling $\bar{\lambda} \approx 16.63$ appears to be somewhat too large to fit the QCD string tension with the leading-order result (6) and also the large- $N_{c}$ lattice result Ref. [30] for $m_{\rho} / \sqrt{\sigma}$. Using the latter as input corresponds to $\bar{\lambda} \approx 12.55(80)$ and gives an almost perfect prediction of $\Gamma_{\rho} / m_{\rho}=0.203$ (14) (if one ignores that $g_{\rho \pi \pi}$ should actually be even a bit larger because (21) refers to 
massless pions). As suggested in the previous section, we shall also consider the lower value of 12.55 as an alternative choice of $\bar{\lambda}$ in order to set up a theoretical error bar.

Turning now to glueball decay rates, one can proceed in analogy to the calculation of the $\rho$ meson decay into pions [53-55]. A metric fluctuation $\delta g_{m n}\left(x^{\mu}, Z\right)=G(x) H(Z) \epsilon_{m n}$ dual to a glueball mode needs to be normalized such that the corresponding scalar, vector, or tensor field $G(x)$ is canonically normalized. This fixes the amplitude of the mode function $H$ such that $H(Z=0)^{-1} \propto \bar{\lambda}^{1 / 2} N_{c} M_{\mathrm{KK}}$. Inserting the metric fluctuations in the D-brane action yields an effective action for the couplings of glueballs to pions and the vector mesons. The resulting general pattern is [53]

$$
\Gamma_{G \rightarrow 2 \pi} / M_{G} \propto \bar{\lambda}^{-1} N_{c}^{-2}, \quad \Gamma_{G \rightarrow 4 \pi} / M_{G} \propto \bar{\lambda}^{-3} N_{c}^{-4},
$$

so that glueballs are predicted as rather narrow states, with the branching ratio into 4 pions being particularly strongly suppressed. A decay into four $\pi^{0}$ is even further reduced [55]: $F^{4}$ terms in the DBI action of the D8 branes give vertices of a glueball with four $\pi^{0}$ such that

$$
\Gamma_{G \rightarrow 4 \pi^{0}} / M_{G} \propto \bar{\lambda}^{-7} N_{c}^{-4},
$$

while nonlinear terms of $G$ in the Yang-Mills part of the DBI action give rise to

$$
\Gamma_{G \rightarrow G+2 \pi^{0} \rightarrow 4 \pi^{0}} / M_{G} \propto \bar{\lambda}^{-3} N_{c}^{-6},
$$

the latter with strong kinematical suppression from phase space integrals.

In Ref. [53], the decay rates of the glueball corresponding to the lowest scalar mode has been calculated, which, as discussed above, comes out with a mass $M_{G} \approx 0.9 M_{\mathrm{KK}}$, which is only half of what is expected from lattice calculations, and which appears exotic in that it involves a metric polarization $g_{44}$. In Ref. $[54,55]$ we have considered also the scalar and tensor glueball associated with transverse-traceless modes in $\mathrm{AdS}_{7} \times S^{4}$, which provide glueballs of mass $M_{G} \approx 1.567 M_{\mathrm{KK}} \approx$ $1487 \mathrm{MeV}$ and thus not far from the lowest glueball in lattice simulations. The coupling of this glueball to two pions turns out to read

$$
S_{G(1487) \rightarrow \pi \pi}=\int d^{4} x \frac{1}{2} \tilde{c}_{1} \partial_{\mu} \pi^{a} \partial_{v} \pi^{a}\left(\eta^{\mu \nu}-\frac{\partial^{\mu} \partial^{v}}{M_{G}^{2}}\right) G,
$$

with $\tilde{c}_{1}=17.23 \bar{\lambda}^{-1 / 2} N_{c}^{-1} M_{\mathrm{KK}}^{-1}$, which yields

$$
\frac{\Gamma_{G(1487) \rightarrow \pi \pi}}{M_{G}}=\frac{3\left|\tilde{c}_{1}\right|^{2} M_{G}^{2}}{512 \pi}=\frac{1.359}{\bar{\lambda} N_{c}^{2}}=\left\{\begin{array}{l}
0.009 \\
0.012
\end{array} \text { for } \bar{\lambda}=\left\{\begin{array}{l}
16.63 \\
12.55
\end{array} .\right.\right.
$$

This result is significantly smaller than the relative width obtained for the lowest "exotic" scalar mode (which is of the same parametric order but numerically has a larger coupling ${ }^{10}$ ), suggesting that the scalar glueball with $M=1487 \mathrm{MeV}$ does not behave like an excited scalar, which one would expect to have a larger decay width. This adds to the suspicion that the lowest "exotic" scalar might have to be discarded.

The prediction of the Witten-Sakai-Sugimoto model is then a scalar glueball of roughly the mass found in lattice calculations and with a very narrow width. This should be contrasted with the findings of Ref. [57], where an extended linear sigma model with a dilaton describing a narrow glueball could only be accommodated with an uncomfortably large gluon condensate. In the Witten-Sakai-Sugimoto model a narrow glueball seems to be perfectly compatible with a comparatively small gluon condensate (16).

\footnotetext{
${ }^{10}$ The relative width has been obtained as 0.040 for $\bar{\lambda}=16.63$ in Ref. [53], but according to [55] the effective action derived in Ref. [53] is incomplete, and the resulting relative width of the lowest "exotic" scalar mode is even larger, namely 0.092.
} 


\section{Deconfinement, chiral symmetry restoration, and the effects of strong magnetic fields}

\subsection{Deconfinement transition to black D4 phase and chiral symmetry restoration}

The Witten-Sakai-Sugimoto model can be extended to finite temperature $T$ by compactifying also imaginary time $i x^{0}$ with a period $\beta=1 / T$. Periodic boundary conditions for bosons and antiperiodic boundary conditions for fermions give the correct statistics for the thermodynamic partition function, which breaks supersymmetry spontaneously exactly as the spatial circle in $x_{4}$ does. For low temperature (large $\beta$ ), the gravitational background is just (3) with imaginary time and $i x^{0} \simeq i x^{0}+\beta$. As the temperature increases, the thermal circle shrinks and a phase transition happens when the circumference of the thermal circle becomes equal to that of the $x_{4}$-circle, because at temperatures higher than that it is energetically favorable that the Euclidean black hole with its cigar topology in the $x_{4}-u$ subspace exchanged with the cylindrical topology in the $i x^{0}-u$ subspace [48]. The phase transition temperature is simply given by

$$
\beta=L_{4} \equiv 2 \pi R_{4} \Leftrightarrow T_{c}=\frac{1}{2 \pi} M_{\mathrm{KK}} .
$$

This corresponds to the appearance of an actual black hole (more precisely a black 4-brane) in the real-time geometry, analogous to a Hawking-Page transition [5]. Since with this swapping of the roles of $x_{4}$ and $i x^{0}$, the blackening function $f(u)$ now appears in $g_{t t}$, with $u_{\mathrm{KK}}$ replaced by $u_{T}=(4 \pi T / 3)^{2} R^{3}$, and the string tension (6) suddenly becomes zero, signalling deconfinement.

Unfortunately, this deconfinement transition is not smoothly connected to the deconfinement transition in the 3+1-dimensional Yang-Mills theory and we shall mention an alternative proposal for a more adequate holographic model below. The deconfined phase corresponding to the black 4-brane is nevertheless interesting, because it gives rise to a nontrivial pattern of chiral symmetry restoration if one relaxes the original setup of antipodal D8-D8-branes [48]. When the latter are maximally separated, as in the original Sakai-Sugimoto model, chiral symmetry is necessarily restored when the cigar topology in the $u-x_{4}$ is replaced by a cylindrical topology. The connection between the D8- $\overline{\mathrm{D} 8}$ branes is broken and their configuration becomes that of simple straight embeddings down to $u=u_{T}$, the black brane horizon. However, with nonmaximal separation, the $\mathrm{D} 8-\overline{\mathrm{D}} 8$-branes can connect also within the cylindrical topology, and it depends on the free energy encoded in the D8-action which configuration is favored. It turns out that for brane separation $L<0.97 R_{4}$ there is still a chiral symmetry breaking phase when the deconfined phase is entered. If one also introduces a chemical potential for quark number in the form of an asymptotic value of the $\mathrm{U}(1)$-component of $A^{0}$, the zeroth component of the flavor gauge field, the phase diagrams takes the form displayed in Fig. 3 [58].

This phase diagram has also been studied in the presence of baryonic matter represented by sources at the tip of the D8 branes [59], and refinements involving extended, non-point-like baryons have been studied in [60-63]. Also a very interesting attempt [64] has been made to find a holographic realization of the so-called quarkyonic phase $[65,66]$ within the confined phase.

\subsection{Deconfinement through a Gregory-Laflamme transition}

The first-order transition to the black 4-brane phase takes place at a temperature (27) directly set by $M_{\mathrm{KK}}: T_{c}=M_{\mathrm{KK}} / 2 \pi \approx 151 \mathrm{MeV}$. Superficially, this looks appealing as the (crossover) temperature for deconfinement and chiral symmetry breaking of real QCD is indeed close to that value. However, when compared to the first-order phase transition temperature for pure-glue (or quenched) QCD, it is uncomfortably small. 


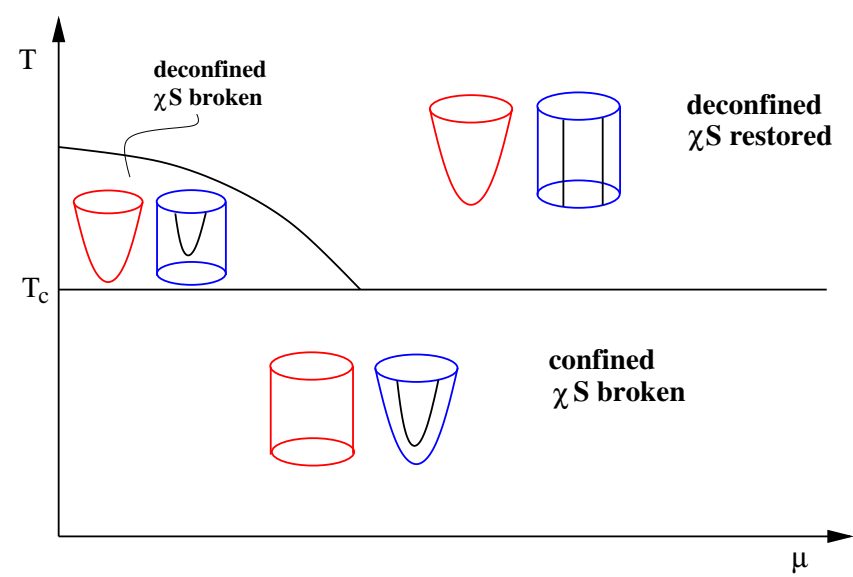

Figure 3. Phase diagram of the Sakai-Sugimoto model where the deconfinement transition is realized by a Hawking-Page transition. The topology of the $i x^{0}-u$ and $x_{4}-u$ subspaces is sketched in red and blue, respectively, with the D8- $\overline{\mathrm{D} 8}$-brane configuration drawn in black.

A more important issue with this deconfinement transition has been noted first in Ref. [48]: In the limit of small 't Hooft coupling and large $M_{\mathrm{KK}}$, which would be required to actually reach 3+1dimensional Yang-Mills theory, the critical temperature will have to occur at $T \sim \Lambda_{\mathrm{QCD}} \ll M_{\mathrm{KK}}=$ $R_{4}^{-1}$. However, due to the symmetry under $\beta \leftrightarrow L_{4} \equiv 2 \pi R_{4}$, there will then also be a phase transition at a temperature $T \gg R_{4}^{-1}$. The phase transition line given by (27), if it connects to the deconfinement of 3+1-dimensional Yang-Mills theory, needs to bifurcate at some value of $\lambda_{5} / R_{4}$; it cannot smoothly connect.

In Ref. [67] it has recently been pointed out that the $Z_{N_{c}} \times Z_{N_{c}}$ center symmetry for the two circle compactifications along $i x^{0}$ and $x_{4}$ matches the phases of 3+1-dimensional Yang-Mills theory only in the low-temperature configuration, but not in the high-temperature, black 4-brane geometry. Hence, there definitely has to be a phase transition between the latter and actual deconfined 3+1-dimensional Yang-Mills theory. The authors of Ref. [67] have argued that one should instead consider a phase transition with periodic instead of antiperiodic boundary conditions for fermions on the thermal circle. This is not appropriate for fermions, but the target of the Witten model is pure Yang-Mills theory without fermions anyway. In this case, the symmetry $\beta \leftrightarrow L_{4}$ is avoided and the gravity dual of the deconfinement transition is a Gregory-Laflamme transition into the T-dual type IIB supergravity, where the D4 branes are replaced by an inhomogeneous distribution of D3 branes. Then the center symmetry breaking is such that it does not rule out a smooth connection to the deconfinement transition in 3+1-dimensional Yang-Mills theory.

Using prior work on the Gregory-Laflamme instability of branes compactified on a circle [69], Ref. [67] has obtained the following estimate for the first-order transition temperature:

$$
T_{\mathrm{GL}} \simeq \frac{\bar{\lambda}}{2 \pi \cdot 8.54} M_{\mathrm{KK}}
$$

Inserting our fit parameters, this gives $T_{\mathrm{GL}}=294$ (222) $\mathrm{MeV}$ for $\bar{\lambda}=16.63$ (12.55), indeed a quantatively more appealing ballpark for the deconfinement temperature of quenched QCD than the transition temperature (27) with antiperiodic boundary conditions on the thermal circle. For the fermions that are introduced through probe flavor branes, Ref. [67] suggested to use an imaginary chemical 


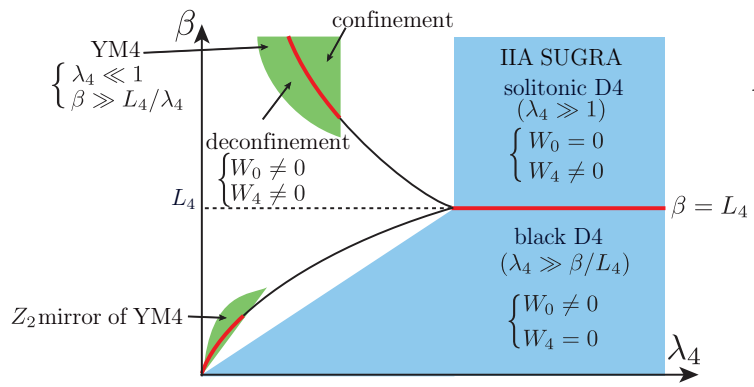

$(\mathrm{AP}, \mathrm{AP})$

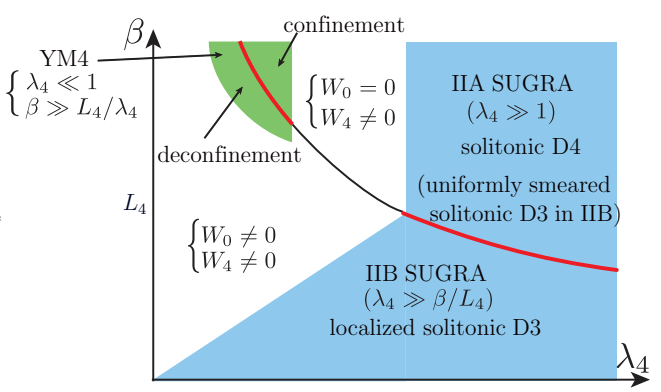

$(\mathrm{P}, \mathrm{AP})$

Figure 4. Deconfinement phase transitions with antiperiodic and periodic boundary conditions along the thermal circle, with $W_{0}$ and $W_{4}$ referring to the expection values of Polyakov loops in temporal and $x_{4}$ direction, respectively (taken from Ref. [68]).

potential to get the statistics right, but the details of this alternative proposal for a deconfined WittenSakai-Sugimoto model have still to be worked out.

\subsection{The effects of strong magnetic fields on chiral symmetry breaking and restoration}

While the simple deconfinement transition based on black 4-branes appears to be lacking important features of the 3+1-dimensional deconfinement of nonsupersymmetric Yang-Mills theory, it may nevertheless be interesting to study the chiral phase transition in the simple background of black 4-branes. With nonmaximal separation of the D8 branes, the chiral phase transition is moved away from the deconfinement transition. For sufficiently small D8 brane separation, the deconfinement temperature can actually be sent to arbitarily small temperature [70]. In this limit, the Sakai-Sugimoto model actually becomes dual to a nonlocal NJL model, as has been pointed out in Ref. [47], and the phase diagram in the $T$ - $\mu$-plane is similar to that found in conventional NJL models.

With nonantipodal D8 branes, the phase diagram moreover becomes sensitive to external fields, which makes this setup an interesting toy model for exploring possible phenomena related to strong electromagnetic fields, in particular their impact on chiral symmetry breaking. At zero baryon chemical potential, magnetic catalysis of chiral symmetry breaking was shown to occur in the SakaiSugimoto model [71, 72], in accordance with the field-theoretic analysis of Ref. [73]. At low temperature and high chemical potential, an opposite effect ("inverse magnetic catalysis") was observed in Ref. $[74,75]$ that has to do with an additional phase transition $[74,76]$ that can be interpreted as a transition into the lowest Landau level (LLL) of chiral quarks (Fig. 5). ${ }^{11}$ Indeed, a very similar phase diagram was previously found in NJL models $[78,79]$. In contrast to the latter, the holographic model does not show de Haas-van Alphen oscillations, which indicates that in the holographic model there is no sharp Fermi surface (see also Refs. [80, 81]).

An interesting feature, shared with real QCD [82], is that in the chirally broken phase at finite chemical potential and with a background magnetic field, baryon number is generated through the axial anomaly by gradients of the pion field. On the holographic side, this is brought about by the Chern-Simons part of the D8 brane action [83-85]. ${ }^{12}$

\footnotetext{
${ }^{11}$ At zero chemical potential, the magnetic field monotonically increases the critical temperature for chiral symmetry restoration, signalling ordinary magnetic catalysis, in contrast to the recent lattice results for QCD at physical quark masses [77].

${ }^{12}$ There is however a still unresolved issue with boundary terms that need to be added to the action to insure thermodynamic consistency, which comes at the price of modifying the anomaly content [84-86].
} 


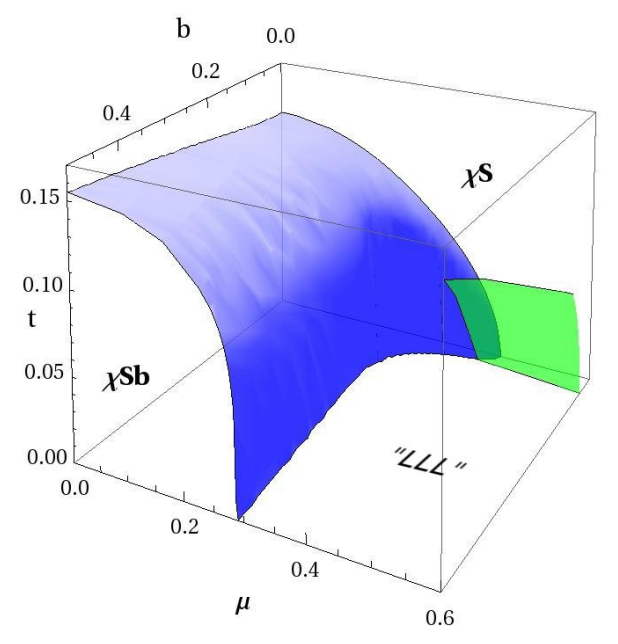

Figure 5. Phase diagram of the Sakai-Sugimoto model in (dimensionless) temperature $(t)$, quark chemical potential $(\mu)$, and magnetic field strength at small D8 brane separation such that the deconfinement transition is at $t \approx 0$, without baryonic matter from D4 branes [74] (the effects of including the latter have been discussed in [87]). At small $\mu$, the critical temperature for chiral symmetry restoration rises with $b$ (normal magnetic catalysis), whereas at low $T$, the dependence is nonmonotonic. Beyond a critical temperature, there is also a magnetic phase transition (green surface) into a "lowest Landau-level" phase.

Another consequence of the axial anomaly is the appearance of anomalous nondissipative transport phenomena. In the Sakai-Sugimoto model at finite chemical potential and nonzero magnetic field, the chirally symmetric phase carries an axial current $\mathbf{j}_{A} \propto \mu \mathbf{B}$ in accordance with the field-theoretic result obtained in Ref. [88, 89].

A related effect is the so-called chiral magnetic effect $[90,91]$ that has attracted a lot of attention because it may be studied in heavy-ion collisions where sufficiently strong magnetic fields could arise in noncentral collisions. Here an imbalance between left and right chiral fermions, described by an axial chemical potential, gives rise to a vector current and electric charge separation through $\mathbf{j}_{V} \propto \mu_{A} \mathbf{B}$ as first noted in a different context in Ref. [92] and independently rediscovered by various authors. In the Sakai-Sugimoto model this effect was reproduced in Ref. [93], including a calculation of the frequency dependent chiral conductivity, but it involves some conceptual issues [86] that were clarified only recently $[94,95]$.

\section{Conclusion}

The Witten-Sakai-Sugimoto model is certainly the best studied and most developed top-down holographic approach to a nonsupersymmetric nonconformal Yang-Mills theory at strong coupling. As we have discussed, this model reproduces many phenomena that are known to characterize low-energy QCD such as confinement, chiral symmetry breaking, mesons including glueballs, baryons, and effects from the axial anomaly. Although this model is actually a 4+1-dimensional super-Yang-Mills theory above a Kaluza-Klein scale that cannot be made much larger than $1 \mathrm{GeV}$ without leaving the supergravity approximation, extending the results of these calculations to finite $N_{c}=3$ and a finite 't Hooft coupling does give a surprisingly good fit of most of the quantities that can be compared with experiment or controllable lattice calculations. Parametrically, the predictions are in line with the expectations from large- $N_{c}$ analysis, but, optimistically, they also give a rough estimate of the concrete magnitude of various effects. For example, glueball decay rates are found to be numerically very small, which is an interesting prediction given that the decay rates of known mesons are quite well reproduced by this model. This may motivate the inclusion of this and related predictions in other, phenomenological models, if only as a ballpark estimate. Similarly, the model may serve to give thought-provoking suggestions for the phase diagram of QCD in regions which will hopefully be explored by first-principle lattice simulations. Indeed, it seems that the notorious sign problem of 
lattice QCD at finite chemical potential could be overcome in the future by new ingenious techniques [96].

On the other hand, the Witten-Sakai-Sugimoto model, being also a first-principle approach, will hopefully also see further developments. Important and ambitious directions would be the inclusion of backreaction of flavor branes, corresponding to an unquenching of the quarks [27, 28, 97], an improved description of the deconfined phase [68], the inclusion of finite quark masses [98-100], or the inclusion of string-theoretic corrections.

\section{Acknowledgements}

I would like to thank Frederic Brünner, Denis Parganlija, Florian Preis, Andreas Schmitt, and Stefan Stricker for enjoyable collaborations on the Witten-Sakai-Sugimoto model as well as Matthias Lutz, Marco Panero and Shigeki Sugimoto for discussions. I am particularly grateful to Frederic Brünner, Denis Parganlija, and Florian Preis for a careful reading of this manuscript, and to Andreas Schmitt for some of the illustrations.

\section{References}

[1] M. Lutz et al. (PANDA Collaboration) (2009), 0903 . 3905

[2] U. Wiedner, Prog.Part.Nucl.Phys. 66, 477 (2011), 1104 . 3961

[3] B. Friman, C. Hohne, J. Knoll, S. Leupold, J. Randrup et al., Lect.Notes Phys. 814, 1 (2011)

[4] O. Aharony, S.S. Gubser, J.M. Maldacena, H. Ooguri, Y. Oz, Phys.Rept. 323, 183 (2000), hep-th/9905111

[5] E. Witten, Adv.Theor.Math.Phys. 2, 505 (1998), hep-th/9803131

[6] T. Sakai, S. Sugimoto, Prog.Theor.Phys. 113, 843 (2005), hep-th/0412141

[7] T. Sakai, S. Sugimoto, Prog.Theor.Phys. 114, 1083 (2005), hep-th/0507073

[8] R.C. Myers, M.F. Paulos, A. Sinha, Phys.Rev. D79, 041901 (2009), 0806.2156

[9] J.P. Blaizot, E. Iancu, U. Kraemmer, A. Rebhan, JHEP 0706, 035 (2007), hep-ph/0611393

[10] D. Kharzeev, K. Landsteiner, A. Schmitt, H.U. Yee, Lect.Notes Phys. 871, 1 (2013)

[11] K. Peeters, M. Zamaklar, Eur.Phys.J.ST 152, 113 (2007), 0708. 1502

[12] G. 't Hooft, Nucl.Phys. B72, 461 (1974)

[13] J.M. Maldacena, Int.J.Theor.Phys. 38, 1113 (1999), hep-th/9711200

[14] L. Susskind, J.Math.Phys. 36, 6377 (1995), hep-th/9409089

[15] G. Policastro, D.T. Son, A.O. Starinets, Phys.Rev.Lett. 87, 081601 (2001), hep-th/0104066

[16] P. Kovtun, D.T. Son, A.O. Starinets, Phys.Rev.Lett. 94, 111601 (2005), hep-th/0405231

[17] J. Casalderrey-Solana, D.C. Gulhan, J.G. Milhano, D. Pablos, K. Rajagopal, Nucl.Phys. A (2014), 1408.5616

[18] E. Iancu, A. Mukhopadhyay (2014), 1410.6448

[19] P.G. Freund, M.A. Rubin, Phys.Lett. B97, 233 (1980)

[20] I. Kanitscheider, K. Skenderis, M. Taylor, JHEP 0809, 094 (2008), 0807 . 3324

[21] R.C. Brower, S.D. Mathur, C.I. Tan, Nucl.Phys. B587, 249 (2000), hep-th/0003115

[22] B. Lucini, A. Rago, E. Rinaldi, JHEP 1008, 119 (2010), 1007. 3879

[23] C. Csaki, H. Ooguri, Y. Oz, J. Terning, JHEP 9901, 017 (1999), hep-th/9806021

[24] C. Csaki, J. Russo, K. Sfetsos, J. Terning, Phys.Rev. D60, 044001 (1999), hep-th/9902067

[25] N.R. Constable, R.C. Myers, JHEP 9910, 037 (1999), hep-th/9908175

[26] M. Kruczenski, D. Mateos, R.C. Myers, D.J. Winters, JHEP 0405, 041 (2004), hep-th/0311270 
[27] B.A. Burrington, V.S. Kaplunovsky, J. Sonnenschein, JHEP 0802, 001 (2008), 0708. 1234

[28] F. Bigazzi, A.L. Cotrone (2014), 1410.2443

[29] J.L. Barbon, C. Hoyos-Badajoz, D. Mateos, R.C. Myers, JHEP 0410, 029 (2004), hep-th/0404260

[30] G.S. Bali, F. Bursa, L. Castagnini, S. Collins, L. Del Debbio et al., JHEP 1306, 071 (2013), 1304.4437

[31] M.J. Teper (1997), hep-lat/9711011

[32] M.A. Shifman, A. Vainshtein, V.I. Zakharov, Nucl.Phys. B147, 385 (1979)

[33] B. Ioffe, Prog.Part.Nucl.Phys. 56, 232 (2006), hep-ph/0502148

[34] A. Samsonov (2004), hep-ph/0407199

[35] S. Narison, Phys.Lett. B706, 412 (2012), 1105.2922

[36] G.S. Bali, C. Bauer, A. Pineda, Phys.Rev.Lett. 113, 092001 (2014), 1403.6477

[37] G. Colangelo, S. Durr, A. Juttner, L. Lellouch, H. Leutwyler et al., Eur.Phys.J. C71, 1695 (2011), 1011.4408

[38] T. Imoto, T. Sakai, S. Sugimoto, Prog.Theor.Phys. 124, 263 (2010), 1005.0655

[39] J. Schechter, H. Weigel (1999), hep-ph/9907554

[40] E. Witten, JHEP 9807, 006 (1998), hep-th/9805112

[41] D. Son, M. Stephanov, Phys.Rev. D69, 065020 (2004), hep-ph/0304182

[42] D.K. Hong, M. Rho, H.U. Yee, P. Yi, Phys.Rev. D76, 061901 (2007), hep-th/0701276

[43] H. Hata, T. Sakai, S. Sugimoto, S. Yamato, Prog.Theor.Phys. 117, 1157 (2007), hep-th/0701280

[44] K. Hashimoto, T. Sakai, S. Sugimoto, Prog.Theor.Phys. 120, 1093 (2008), 0806. 3122

[45] S. Seki, J. Sonnenschein, JHEP 0901, 053 (2009), 0810. 1633

[46] G.S. Adkins, C.R. Nappi, E. Witten, Nucl.Phys. B228, 552 (1983)

[47] E. Antonyan, J. Harvey, S. Jensen, D. Kutasov (2006), hep-th/0604017

[48] O. Aharony, J. Sonnenschein, S. Yankielowicz, Annals Phys. 322, 1420 (2007), hep-th/0604161

[49] N. Callebaut, D. Dudal, H. Verschelde, JHEP 1303, 033 (2013), 1105. 2217

[50] H. Boschi-Filho, N.R. Braga, Eur.Phys.J. C32, 529 (2004), hep-th/0209080

[51] P. Colangelo, F. De Fazio, F. Jugeau, S. Nicotri, Phys.Lett. B652, 73 (2007), hep-ph/0703316

[52] H. Forkel, Phys.Rev. D78, 025001 (2008), 0711. 1179

[53] K. Hashimoto, C.I. Tan, S. Terashima, Phys.Rev. D77, 086001 (2008), 0709. 2208

[54] F. Brünner, D. Parganlija, A. Rebhan, Acta Phys.Polon.Supp. 7, 533 (2014), 1407.6914

[55] F. Brünner, D. Parganlija, A. Rebhan (2014), in preparation

[56] K. Olive et al. (Particle Data Group), Chin.Phys. C38, 090001 (2014)

[57] S. Janowski, F. Giacosa, D.H. Rischke (2014), 1408 . 4921

[58] N. Horigome, Y. Tanii, JHEP 0701, 072 (2007), hep-th/0608198

[59] O. Bergman, G. Lifschytz, M. Lippert, JHEP 0711, 056 (2007), 0708. 0326

[60] K.Y. Kim, S.J. Sin, I. Zahed, JHEP 0809, 001 (2008), 0712 . 1582

[61] M. Rozali, H.H. Shieh, M. Van Raamsdonk, J. Wu, JHEP 0801, 053 (2008), 0708 . 1322

[62] V. Kaplunovsky, D. Melnikov, J. Sonnenschein, JHEP 1211, 047 (2012), 1201.1331

[63] K. Ghoroku, K. Kubo, M. Tachibana, T. Taminato, F. Toyoda, Phys.Rev. D87, 066006 (2013), 1211.2499 
[64] J. de Boer, B.D. Chowdhury, M.P. Heller, J. Jankowski, Phys.Rev. D87, 066009 (2013), 1209.5915

[65] L. McLerran, R.D. Pisarski, Nucl.Phys. A796, 83 (2007), 0706. 2191

[66] L.Y. Glozman, R. Wagenbrunn, Phys.Rev. D77, 054027 (2008), 0709. 3080

[67] G. Mandal, T. Morita, JHEP 1109, 073 (2011), 1107.4048

[68] G. Mandal, T. Morita, J.Phys.Conf.Ser. 343, 012079 (2012), 1111.5190

[69] T. Harmark, N.A. Obers, JHEP 0409, 022 (2004), hep-th/0407094

[70] A. Parnachev, D.A. Sahakyan, Phys.Rev.Lett. 97, 111601 (2006), hep-th/0604173

[71] C.V. Johnson, A. Kundu, JHEP 0812, 053 (2008), 0803.0038

[72] O. Bergman, G. Lifschytz, M. Lippert, JHEP 0805, 007 (2008), 0802 . 3720

[73] V. Gusynin, V. Miransky, I. Shovkovy, Phys.Lett. B349, 477 (1995), hep-ph/9412257

[74] F. Preis, A. Rebhan, A. Schmitt, JHEP 1103, 033 (2011), 1012. 4785

[75] F. Preis, A. Rebhan, A. Schmitt, Lect.Notes Phys. 871, 51 (2013), 1208.0536

[76] G. Lifschytz, M. Lippert, Phys.Rev. D80, 066007 (2009), 0906. 3892

[77] G. Bali, F. Bruckmann, G. Endrodi, Z. Fodor, S. Katz et al., JHEP 1202, 044 (2012), 1111.4956

[78] D. Ebert, K. Klimenko, M. Vdovichenko, A. Vshivtsev, Phys.Rev. D61, 025005 (2000), hep-ph/9905253

[79] T. Inagaki, D. Kimura, T. Murata, Prog.Theor.Phys. 111, 371 (2004), hep-ph/0312005

[80] M. Kulaxizi, A. Parnachev, Nucl.Phys. B815, 125 (2009), 0811. 2262

[81] B.S. DiNunno, M. Ihl, N. Jokela, J.F. Pedraza, JHEP 1404, 149 (2014), 1403 . 1827

[82] D. Son, M. Stephanov, Phys.Rev. D77, 014021 (2008), 0710. 1084

[83] E.G. Thompson, D.T. Son, Phys.Rev. D78, 066007 (2008), 0806.0367

[84] O. Bergman, G. Lifschytz, M. Lippert, Phys.Rev. D79, 105024 (2009), 0806.0366

[85] A. Rebhan, A. Schmitt, S.A. Stricker, JHEP 0905, 084 (2009), 0811. 3533

[86] A. Rebhan, A. Schmitt, S.A. Stricker, JHEP 1001, 026 (2010), 0909. 4782

[87] F. Preis, A. Rebhan, A. Schmitt, J.Phys. G39, 054006 (2012), 1109.6904

[88] M.A. Metlitski, A.R. Zhitnitsky, Phys.Rev. D72, 045011 (2005), hep-ph/0505072

[89] G. Newman, D. Son, Phys.Rev. D73, 045006 (2006), hep-ph/0510049

[90] K. Fukushima, D.E. Kharzeev, H.J. Warringa, Phys.Rev. D78, 074033 (2008), 0808 . 3382

[91] D.E. Kharzeev, Prog.Part.Nucl.Phys. 75, 133 (2014), 1312 . 3348

[92] A. Vilenkin, Phys.Rev. D22, 3080 (1980)

[93] H.U. Yee, JHEP 0911, 085 (2009), 0908 . 4189

[94] A. Gynther, K. Landsteiner, F. Pena-Benitez, A. Rebhan, JHEP 1102, 110 (2011), 1005. 2587

[95] A. Jimenez-Alba, K. Landsteiner, L. Melgar (2014), 1407. 8162

[96] G. Aarts, E. Seiler, D. Sexty, I.O. Stamatescu (2014), 1408. 3770

[97] J. Erdmenger, V.G. Filev, D. Zoakos, JHEP 1208, 004 (2012), 1112.4807

[98] O. Bergman, S. Seki, J. Sonnenschein, JHEP 0712, 037 (2007), 0708. 2839

[99] A. Dhar, P. Nag, JHEP 0801, 055 (2008), 0708 . 3233

[100] K. Hashimoto, T. Hirayama, F.L. Lin, H.U. Yee, JHEP 0807, 089 (2008), 0803.4192 